\title{
Deracemization of a Racemic Allylic Sulfoxide Using Viedma Ripening
}

\author{
Anthonius H. J. Engwerda, ${ }^{\dagger}$ Niels Koning, ${ }^{\dagger}$ Paul Tinnemans, ${ }^{\dagger}$ Hugo Meekes, ${ }^{*}{ }^{\dagger}$ \\ F. Matthias Bickelhaupt, ${ }^{\dagger,+(0)}$ Floris P. J. T. Rutjes, ${ }^{\dagger}{ }^{\circ}$ and Elias Vlieg ${ }^{\dagger}$ (1)
}

${ }^{\dagger}$ Institute for Molecules and Materials, Radboud University, Heyendaalseweg 135, 6525 AJ Nijmegen, The Netherlands

${ }^{\ddagger}$ Department of Theoretical Chemistry and Amsterdam Center for Multiscale Modeling, VU University, De Boelelaan 1083, 1081 HV Amsterdam, The Netherlands

Supporting Information

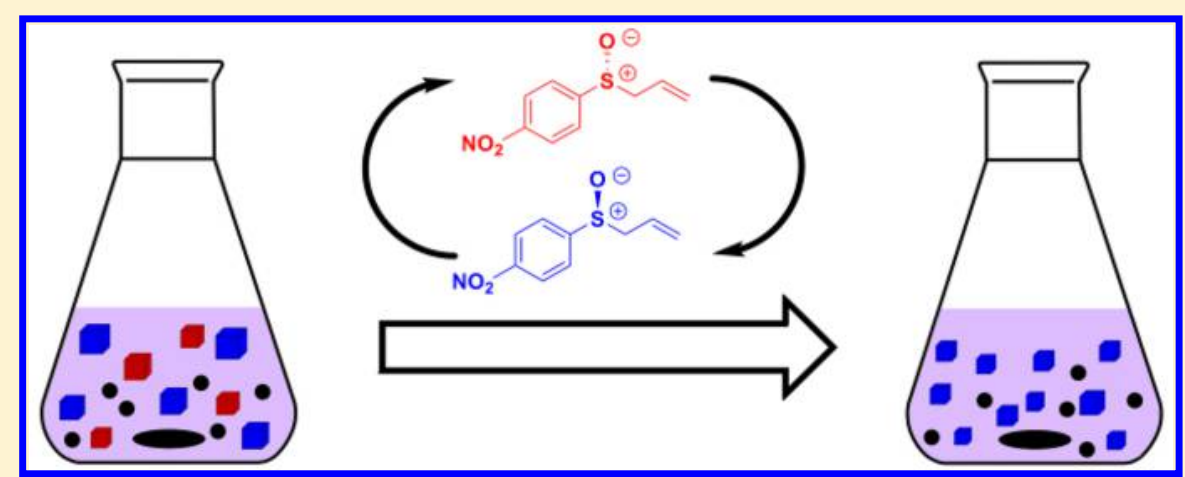

ABSTRACT: Despite the importance of enantiopure chiral sulfoxides, few methods exist that allow for their deracemization. Here, we show that an enantiopure sulfoxide can be produced from the corresponding racemate using Viedma ripening involving rearrangement-induced racemization. The suitable candidate for Viedma ripening was identified from a library of 24 chiral sulfoxides through X-ray structure determination. Starting from the racemic sulfoxide, an unprecedented application of a 2,3sigmatropic rearrangement type racemization in a Viedma ripening process allowed for complete deracemization.

\section{INTRODUCTION}

Single chirality is a key feature of life and hence of utmost importance in the production of active pharmaceutical ingredients in the pharmaceutical industry. In the case of chiral drugs, it is generally one enantiomer that has the desired physiological effects, whereas its mirror image is inactive or even has harmful consequences. ${ }^{1}$ An important class of drugs consists of chiral sulfoxides, where a sulfur instead of a carbon atom is the tetrahedral center of chirality. The best-known example of a chiral sulfoxide is probably esomeprazole, the number two selling drug in 2013 in the United States (marketed as Nexium). This drug acts as a proton pump inhibitor, reducing stomach acid production. ${ }^{2}$ Esomeprazole is an enantiopure sulfoxide that is produced on an industrial scale by asymmetric oxidation of the corresponding sulfide. ${ }^{3}$ It contains only the $(S)$-enantiomer and was found to be more active than the racemate, known as omeprazole. ${ }^{4,5}$ Obtaining an enantiopure compound, however, is generally not straightforward, since (achiral) synthesis will yield both enantiomers in equal amounts, while enantioselective methods in many cases cannot be easily applied. In the majority of cases, resolution using diasteriomeric salt formation is applied to obtain the desired enantiomer, which, however, results in a maximum yield of $50 \%$. As an alternative, a deracemization method might be applied, allowing yields of a single enantiomer in up to $100 \%$ yield. ${ }^{6}$ Viedma ripening is such a method that allows the conversion of a racemic mixture of solids into a single enantiomer in theoretically $100 \%$ yield. ${ }^{7,8}$ It involves grinding of a suspension of racemic conglomerate crystals in combination with racemization in solution, resulting in complete deracemization of the solid phase. The scope of Viedma ripening, however, has so far remained limited due to the lack of suitable racemization strategies. ${ }^{9}$ The majority of examples consist of base-induced racemization through deprotonation, or involve a redox reaction. Consequently, examples of molecules that have been deracemized with Viedma ripening include amino acids ${ }^{10}$ and derivatives, ${ }^{11}$ and metalorganic complexes. ${ }^{12}$ In addition, the group of Håkansson demonstrated Viedma ripening and successive oxidation on a ruthenium-sulfide complex. ${ }^{13}$ However, due to the limitations in racemization methods, and despite the relevance of chiral sulfoxides in drugs, no such molecules have yet been deracemized using Viedma ripening. The purpose of our study is therefore to expand the scope of Viedma ripening to this important class of chiral molecules. In this article, we

Received: June 14, 2017

Published: July 3, 2017 
describe the route that we used to screen for a suitable sulfoxide and disclose the first example of deracemization of a sulfoxide using Viedma ripening.

\section{RESULTS AND DISCUSSION}

A few challenges need to be faced before this particular case of Viedma ripening can be applied. First, the molecule of interest must be able to undergo racemization in solution. While the majority of sulfoxides racemize at temperatures over $200{ }^{\circ} \mathrm{C}^{14}$ allylic sulfoxides racemize at much lower temperatures (even below $50{ }^{\circ} \mathrm{C}^{15}$ ) since they are prone to undergo a 2,3sigmatropic rearrangement, known as the Mislow-Evans rearrangement (Figure 1). ${ }^{16-18}$ Such an intramolecular rearrangement is unprecedented as the racemization mechanism in Viedma ripening.

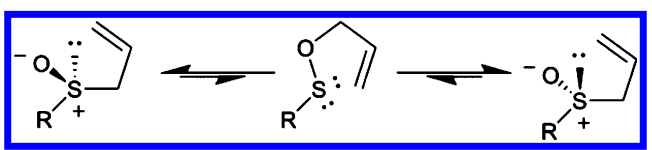

Figure 1. An allylic sulfoxide can reversibly rearrange into an achiral sulfenate via a 2,3-sigmatropic rearrangement, resulting in solution phase racemization of the chiral sulfoxide.

A second requirement for Viedma ripening is that the enantiomers crystallize as separate crystals, i.e., form a racemic conglomerate. For the majority ( $90 \%)$ of compounds, however, the enantiomers crystallize in the same crystal, i.e., form a racemic compound. Thus, the first step in this project is to identify a conglomerate allylic sulfoxide. Since no such conglomerate has been posted in the Cambridge Structural Database (CSD) up to now, we first performed a screening. Salt formation has been described as a route for finding conglomerate crystals. ${ }^{19}$ However, since many sulfoxides are not compatible with salt formation, we decided to engage in a library synthesis.

A series of 24 sulfoxides was synthesized to ensure a reasonable chance of finding a conglomerate (Figure 2, see Supporting Information for a complete overview). To identify sulfoxides displaying conglomerate behavior, crystals suitable for X-ray analysis were grown using slow solvent evaporation. From the 24 synthesized compounds, crystals could be grown of 14 of them, from which we found that compound 1 crystallized as a racemic conglomerate. All other compounds crystallized as racemic compounds (Figure 2).

Compound 1 was subsequently used for the Viedma ripening experiments. The speed of racemization of allylic chiral sulfoxides is highly solvent dependent. ${ }^{20} \mathrm{We}$ found that racemization of compound $\mathbf{1}$ takes place at room temperature in apolar solvents such as toluene and diethyl ether, while the optical purity of the sulfoxide can be retained in polar solvents such as methanol $\left(t_{1 / 2}>4\right.$ days at RT).

By using toluene at room temperature as a solvent and starting from completely racemic conditions, an enantiomeric excess (ee) of $12 \%$ was obtained after a period of over a month. These promising results prompted us to investigate the racemization kinetics, in order to reduce the deracemization time. We found a relatively high barrier for the racemization of $\mathbf{1}$ in toluene at room temperature according to both density functional theory (DFT) calculations $(\Delta H=18.1 \mathrm{kcal} / \mathrm{mol})^{21}$ and temperature dependent selective exchange spectroscopy experiments $(\Delta H=21.2 \mathrm{kcal} / \mathrm{mol})$. This barrier leads to a relatively long racemization half-life $\left(t_{1 / 2}=6.9 \mathrm{~h}\right)$ and results in slow deracemization. We therefore decided to increase the temperature in order to reduce the deracemization time.

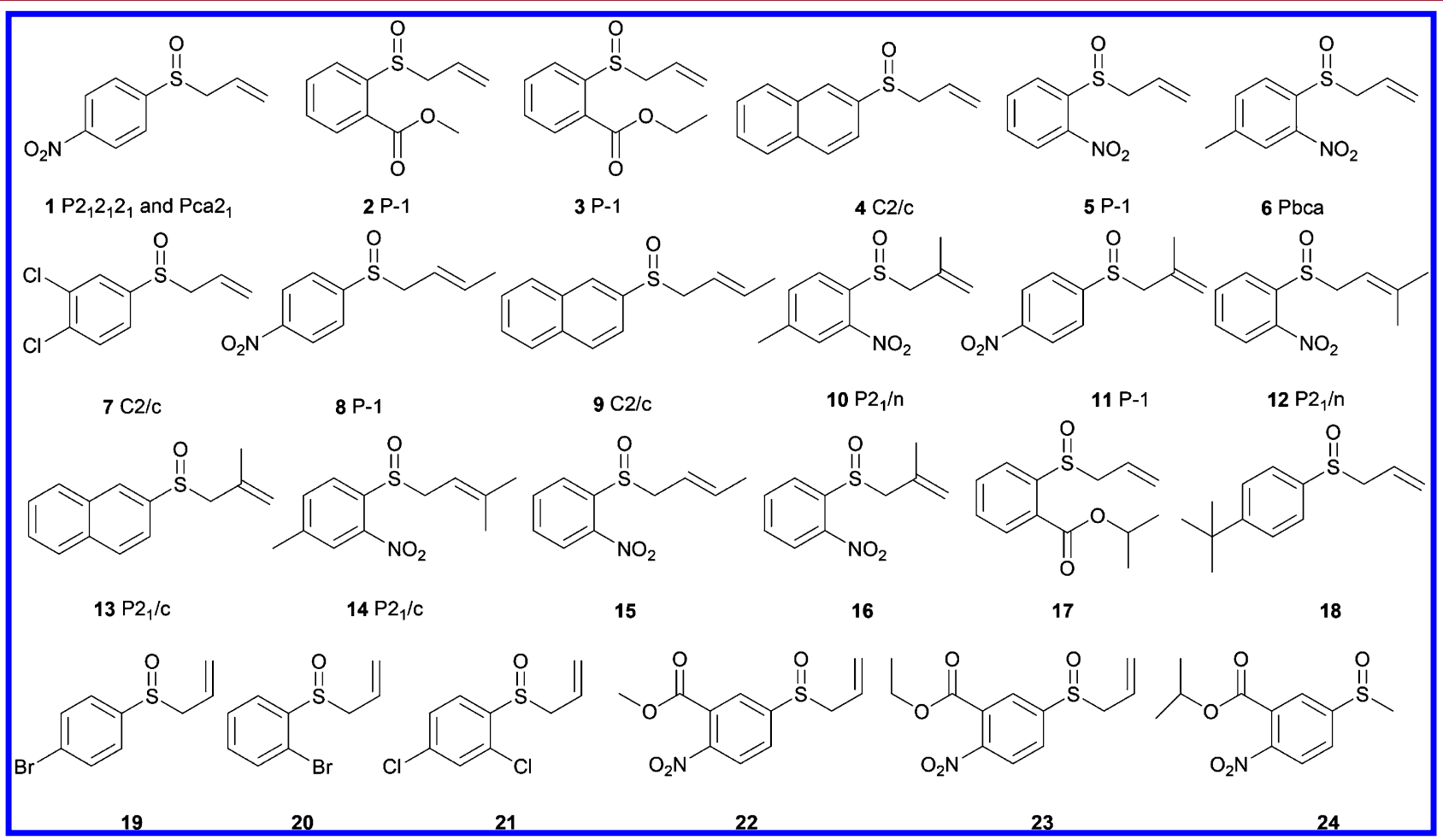

Figure 2. A series of 24 allylic chiral sulfoxides were synthesized, of which crystal structures could be determined for 14 of them (space groups are given after the compound number). Of these 14, only compound 1 crystallized as a racemic conglomerate. 
By changing the solvent system to refluxing diethyl ether $\left(t_{1 / 2}\right.$ $=6.3 \mathrm{~h}$ at $\mathrm{RT}$ and $1.1 \mathrm{~h}$ at reflux, bp $35^{\circ} \mathrm{C}$ ) and creating a small initial bias (around 1\% ee), complete deracemization of 1 could be achieved in just 8 days (Figure 3).

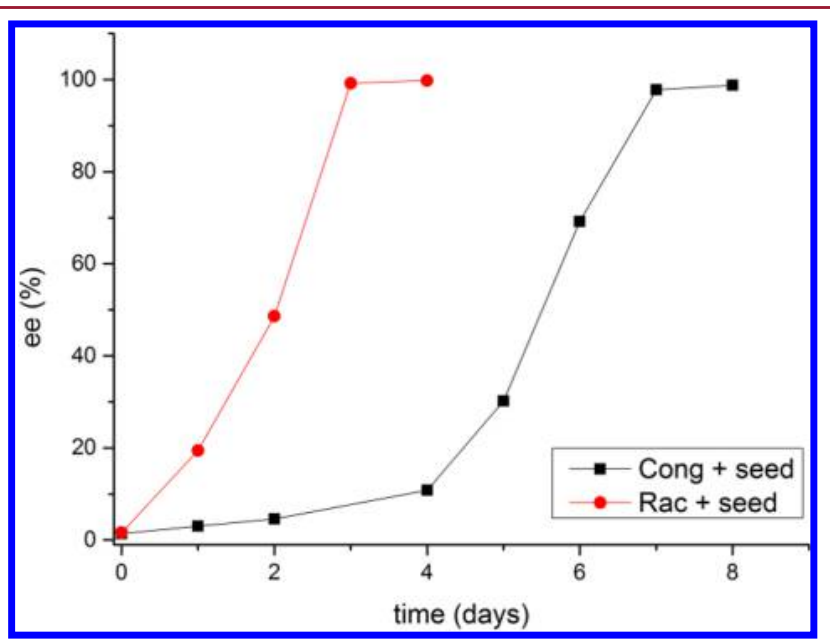

Figure 3. Viedma ripening of compound $\mathbf{1}$ in refluxing diethyl ether starting from the racemic conglomerate compound or metastable racemic compound. In both cases the suspension was seeded with enantiopure crystals.

In order to further decrease the deracemization time, the temperature was further increased to $50{ }^{\circ} \mathrm{C}$. Since this temperature exceeds the boiling point of diethyl ether, the solvent was changed to toluene. Under these conditions, however, deracemization experiments of compound 1 were unsuccessful due to melting of the compound. When the solution was then cooled again, $\mathbf{1}$ recrystallized as a different polymorph with a lower melting point. We found that this low melting polymorph was in fact a metastable racemic compound (see Supporting Information).

We have shown in a previous study that the speed of Viedma ripening can be increased using such a metastable racemic compound in combination with enantiopure seed crystals. ${ }^{22}$ Hence, we investigated whether this same approach could be applied to reduce the deracemization time of chiral sulfoxide $\mathbf{1}$. Indeed, by starting from the racemic compound in combination with enantiopure seed crystals in refluxing diethyl ether, the deracemization time could be further decreased to 3 days (Figures 3 and 4).

\section{CONCLUSION}

We have demonstrated that Viedma ripening can be used to deracemize chiral sulfoxides. By synthesizing 24 chiral allylic sulfoxides which spontaneously racemize through a 2,3signatropic rearrangement at elevated temperatures in solution, one racemic conglomerate compound, suitable for Viedma ripening, was identified. Chiral amplification was achieved for this compound by simply heating a suspension of the molecule under grinding conditions.

This experimentally facile route allows for the straightforward preparation of an enantiopure allylic sulfoxide. That this was done for one compound out of a library of 24 candidates might be considered as a limitation. However, similar enantiopure allylic sulfoxides have been used as chiral pool starting materials, and as chiral auxiliaries for the synthesis of enantiopure acids and esters. ${ }^{23}$ Therefore, one deracemized

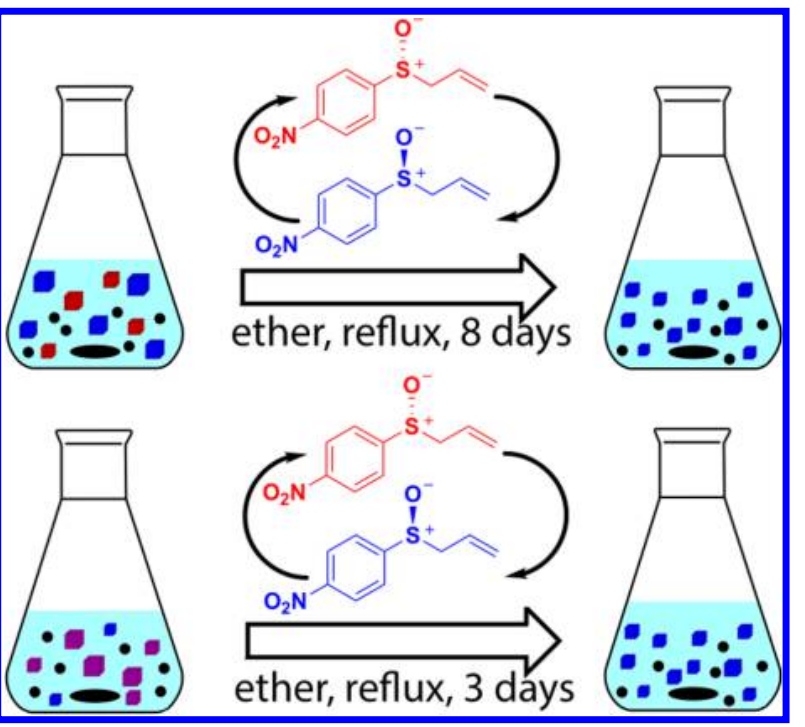

Figure 4. Grinding of a suspension of compound $\mathbf{1}$ in diethyl ether results in complete solid phase deracemization. By performing similar experiments at $50{ }^{\circ} \mathrm{C}$ in toluene, occasional melting and recrystallization resulted in the racemic compound and no deracemization. When grinding a suspension of this racemic compound, seeded with enantiopure crystals in refluxing diethyl ether, fast deracemization could be achieved.

compound can be the starting point for several enantiopure compounds. In addition, the present Viedma ripening screening not only demonstrates the preparation of a specific enantiopure sulfoxide, but also shows a general route that can be followed for the deracemization of other compounds.

\section{ASSOCIATED CONTENT}

\section{S Supporting Information}

The Supporting Information is available free of charge on the ACS Publications website at DOI: 10.1021/acs.cgd.7b00828.

Synthesis and characterization of all compounds, as well as computational details, temperature dependent NMR studies, description of the deracemization experiments and crystal stability experiments (PDF)

\section{Accession Codes}

CCDC 1528086-1528098 and 1528160 contain the supplementary crystallographic data for this paper. These data can be obtained free of charge via www.ccdc.cam.ac.uk/data_request/ cif, or by emailing data_request@ccdc.cam.ac.uk, or by contacting The Cambridge Crystallographic Data Centre, 12 Union Road, Cambridge CB2 1EZ, UK; fax: +44 1223336033.

\section{AUTHOR INFORMATION}

\section{Corresponding Author}

*E-mail: H.Meekes@science.ru.nl.

\section{ORCID $\odot$}

F. Matthias Bickelhaupt: 0000-0003-4655-7747

Floris P. J. T. Rutjes: 0000-0003-1538-3852

Elias Vlieg: 0000-0002-1343-4102

\section{Author Contributions}

A.H.J.E., H.M., F.P.J.T.R. and E.V. designed the experiments and analyzed the data. A.H.J.E. and N.K. performed the experiments. A.H.J.E. and F.M.B. designed the computational experiments. P.T. performed the crystal structure determi- 
nations. A.H.J.E. wrote the manuscript with contributions from all other authors. All authors contributed to the discussions.

Notes

The authors declare no competing financial interest.

\section{ACKNOWLEDGMENTS}

Ing. Erik de Ronde is acknowledged for technical support, Helene Amatdjais-Groenen is acknowledged for assistance with Supercritical fluid chromatography measurements, and Dr. Paul White for assistance with high temperature NMR experiments.

\section{REFERENCES}

(1) Bentley, R. Chem. Soc. Rev. 2005, 34, 609-624.

(2) Li, X. Q.; Andersson, T. B.; Ahlstrom, M.; Weidolf, L. Drug Metab. Dispos. 2004, 32, 821-827.

(3) Olbe, L.; Carlsson, E.; Lindberg, P. Nat. Rev. Drug Discovery 2003, 2, 132-139.

(4) Richter, J. E.; Kahrilas, P. J.; Johanson, J.; Maton, P.; Breiter, J. R.; Hwang, C.; Marino, V.; Hamelin, B.; Levine, J. G. Am. I. Gastroenterol. 2001, 96, 656-665.

(5) Kahrilas, P. J.; Falk, G. W.; Johnson, D. A.; Schmitt, C.; Collins, D. W.; Whipple, J.; D’Amico, D.; Hamelin, B.; Joelsson, B. Studv. Aliment. Pharmacol. Ther. 2000, 14, 1249-1258.

(6) Rachwalski, M.; Vermue, N.; Rutjes, F. P. J. T. Chem. Soc. Rev. 2013, 42, 9268-9282.

(7) Viedma, C. Phvs. Rev. Lett. 2005, 94, 4.

(8) Noorduin, W. L.; Izumi, T.; Millemaggi, A.; Leeman, M.; Meekes, H.; van Enckevort, W. J. P.; Kellogg, R. M.; Kaptein, B.; Vlieg, E.; Blackmond, D. G. I. Am. Chem. Soc. 2008, 130, 1158-1159.

(9) Sogutoglu, L. C.; Steendam, R. R. E.; Meekes, H.; Vlieg, E.; Rutjes, F. P. J. T. Chem. Soc. Rev. 2015, 44, 6723-6732.

(10) Viedma, C.; Ortiz, J. E.; de Torres, T.; Izumi, T.; Blackmond, D. G. I. Am. Chem. Soc. 2008, 130, 15274-15275.

(11) Kaptein, B.; Noorduin, W. L.; Meekes, H.; van Enckevort, W. J. P.; Kellogg, R. M.; Vlieg, E. Angew. Chem., Int. Ed. 2008, 47, 72267229.

(12) Lennartson, A.; Olsson, S.; Sundberg, J.; Håkansson, M. Angew. Chem. Int. Ed. 2009, 48, 3137-3140.

(13) Olsson, S.; Bjoremark, P. M.; Kokoli, T.; Sundberg, J.; Lennartson, A.; McKenzie, C. J.; Håkansson, M. Chem. - Eur. I. 2015, 21, 5211-5219.

(14) Rayner, D. R.; Miller, E. G.; Bickart, P.; Gordon, A. J.; Mislow, K. I. Am. Chem. Soc. 1966, 88, 3138-3139.

(15) Bickart, P.; Carson, F. W.; Jacobus, J.; Miller, E. G.; Mislow, K. I. Am. Chem. Soc. 1968, 90, 4869-4876.

(16) Miller, E. G.; Rayner, D. R.; Mislow, K. I. Am. Chem. Soc. 1966, 88, 3139-3140.

(17) Tang, R.; Mislow, K. L. Am. Chem. Soc. 1970, 92, 2100-2104.

(18) Evans, D. A.; Andrews, G. C.; Sims, C. L. I. Am. Chem. Soc

1971, 93, 4956-4957.

(19) Spix, L.; Alfring, A.; Meekes, H.; van Enckevort, W. J. P.; Vlieg, E. Crvst. Growth Des. 2014, 14, 1744-1748.

(20) Dornan, P. K.; Kou, K. G. M.; Houk, K. N.; Dong, V. M. I. Am. Chem. Soc. 2014, 136, 291-298.

(21) te Velde, G.; Bickelhaupt, F. M.; Baerends, E. J.; Guerra, C. F.; Van Gisbergen, S. J. A.; Snijders, J. G.; Ziegler, T. L. Comput. Chem. 2001, 22, 931-967.

(22) Engwerda, A. H. J.; Meekes, H.; Kaptein, B.; Rutjes, F. P. J. T.; Vlieg, E. Chem. Commun. 2016, 52, 12048-12051.

(23) Hua, D. H.; Venkataraman, S.; Coulter, M. J.; Sinaizingde, G. I. Org. Chem. 1987, 52, 719-728. 